\title{
EFEITO DA CRISE ECONÔMICA NAS FUNÇÕES ORÇAMENTÁRIAS NO SETOR INDUSTRIAL
}

\author{
Ângela Bilk 1 \\ Marcia Zanievicz da Silva ${ }^{2}$ \\ Carlos Eduardo Facin Lavarda ${ }^{3}$
}

- Artigo recebido em: 23/08/2019 -- Artigo aceito em: 19/05/2020 -.' Segunda versão aceita em: 05/02/2021

\section{RESUMO}

O estudo objetivou avaliar a relação de incertezas, em um ambiente de crise econômica, e variáveis endógenas com o uso do orçamento em indústrias catarinenses. Para tal, tem a teoria contingencial como arcabouço teórico. A pesquisa caracteriza-se como descritiva, survey e quantitativa, abrangendo 258 indústrias catarinenses que fazem $\circ$ uso do orçamento como ferramenta gerencial. Constatou-se que a percepção de incerteza em um ambiente de crise econômica se relaciona com o uso do orçamento, modificando o uso das funções orçamentárias. Para as funções de planejamento e alocação de recursos apontou-se uma relação positiva, ou seja, tais funções orçamentárias têm sua relevância ampliada em momentos de crise econômica. De modo contrário, a avaliação de desempenho obteve uma relação negativa, sendo menos utilizada no período de oscilações causadas pela crise econômica. Por fim, conclui-se que no contexto das indústrias catarinenses, as incertezas geradas pelo ambiente de crise econômica, analisadas conjuntamente com variáveis endógenas (estratégia, tamanho e ciclo de vida), modificam as funções orçamentárias. o estudo contribuiu com a pesquisa em contabilidade gerencial ao ampliar o entendimento sobre o uso das funções orçamentárias em períodos de crise econômica.

Palavras-chave: Teoria contingencial. Planejamento. Alocação de recursos. Avaliação de desempenho. Crise econômica.

\footnotetext{
1 Doutoranda em Ciências Contábeis e Administração pela Fundação Universidade Regional de Blumenau - FURB. Professora do Departamento de Ciências Contábeis e Administração da Uniasselvi na Graduação. E-mail: angelabilk07@gmail.com.

https://orcid.org/0000-0003-4321-8547

2 Doutora em Ciências Contábeis e Administração pela Fundação Universidade Regional de Blumenau. Professora da FURB no Programa de Pós-Graduação em Ciências Contábeis. Email: mzsilva@furb.br.

https://orcid.org/0000-0003-1229-7705

3 Doutor em Contabilidade pela Universidade de Valência. Professor do Departamento de Ciências Contábeis da UFSC na Graduação e Pós-Graduação. Bolsista CNPq. E-mail: eduardo.lavarda@ufsc.br.

http://orcid.org/0000-0003-1498-7881
}

Editor responsável pela aprovação do artigo: Dr. Eduardo Mendes Nascimento Editora responsável pela edição do artigo: Drª. Bruna Camargos Avelino 


\title{
EFFECT OF THE ECONOMIC CRISIS ON THE BUDGETARY FUNCTIONS IN THE INDUSTRIAL SECTOR
}

\begin{abstract}
The study aimed at evaluating the relationship of uncertainties, in an environment of economic crisis, and endogenous variables with the use of the budget in Santa Catarina industries. To this end, it has contingency theory as a theoretical framework. The research is characterized as a descriptive, survey, and quantitative, covering 258 industries in Santa Catarina/Brazil that use the budget as a management tool. It was found that the perception of uncertainty in an environment of economic crisis is related to the use of the budget, changing the use of budget functions. A positive relationship was noted for the planning and resource allocation functions, i.e., such budget functions have their relevance expanded in times of economic crisis. On the contrary, performance evaluation has obtained a negative relationship, being less used in the period of oscillations caused by the economic crisis. Finally, we conclude that in the context of Santa Catarina/Brazil industries, the uncertainties generated by the economic crisis environment, analyzed together with endogenous variables (strategy, size, and life cycle), modify the budget functions. The study contributed to the survey in managerial accounting by broadening the understanding of the use of budget functions in periods of economic crisis.
\end{abstract}

Keywords: Contingency theory. Planning. Resource allocation. Performance evaluation. Economic crisis.

\section{INTRODUÇÃO}

Assumindo-se que crises econômicas são decorrentes de um desequilíbrio entre a produção e o consumo (Sandroni, 1994), cuja consequência segundo Becker, Mahlendorf, Schäffer e Thaten (2016), reduz o nível de produção industrial, eleva a taxa de desemprego e provoca alteração nos preços, nos resultados obtidos no período e no volume de investimentos. Nesse contexto, o ambiente afetado por crises econômicas, segundo Asel, Posch e Speckbacher (2011), eleva a incerteza e requer uma redução do tempo hábil para a tomada de decisão, exigindo dos gestores ações imediatas e de curto prazo, capazes de garantir a continuidade da organização. Ainda, Asel et al. (2011) alegam que as crises econômicas aumentam a pressão sobre os gestores no processo de tomada de decisão com o intuito de garantir que as organizações alcancem os objetivos estratégicos estabelecidos.

Como o ambiente afetado por crises econômicas é mais incerto e os objetivos estratégicos tornam-se mais difíceis de serem atingidos, pressupõem-se que as ferramentas que integram o Sistema de Controle Gerencial (SCG) tenham alterações na finalidade de monitorar a gestão, perdendo ou ganhando relevância (Asel et al., 201 1). Possivelmente, a modificação na relevância ou uso das ferramentas gerenciais, em contextos de crises econômicas, decorram da necessidade de readaptar a informação gerada, dada a modificação em atributos como tempestividade e materialidade (Van Der Stede, 2011).

Ademais, em cenários instáveis, como os vivenciados pelo Brasil a partir de 2015, possivelmente aumente a pressão sobre o funcionamento dos sistemas de 
contabilidade gerencial, com intuito de assegurar a continuidade da organização. Nesse sentido, Schaaper, Mizoguchi, Nakamura e Yamashita (2011) evidenciam que o aumento das necessidades informacionais para o processo de tomada de decisão dos gestores, em um ambiente de crises econômicas, pode ser considerado como um fator determinante às mudanças de gestão.

Dentre os diversos tipos de ferramentas gerenciais, o orçamento se destaca; pois, conforme Welsch (1983) e Weston e Brigham (2000), o sistema orçamentário é uma prática importante para a gestão, devido às necessidades de planejamento e de controle, visando a possíveis redirecionamentos para atingir metas e objetivos da organização. Por meio do orçamento, os gestores podem projetar seus gastos e prospectar as fontes de recursos, para posteriormente serem comparados com os dados realizados (Weston \& Brigham, 2000).

O orçamento auxilia as organizações no processo de tomada de decisão, no entanto Hopwood (2009) destaca o uso especificamente em momentos de crises econômicas. De acordo com Leite (2008), a utilização do orçamento tende a aumentar a estimativa dos lucros, bem como o retorno do investimento e, assim, melhorar a situação financeira. Na visão de Frezatti et al. (2010) o uso do orçamento auxilia a gestão organizacional nas suas atividades e prioridades, orientando-os sobre o desempenho, visto que a operacionalização do orçamento acontece por meio do confronto entre o previsto e o realizado, no qual as variações são essenciais e por conseguinte devem ser analisadas e ajustadas para direcionar a organização ao alcance de seus objetivos. Da mesma forma, Panosso et al. (2017) destacam que o emprego do orçamento impacta no desempenho das organizações, uma vez que sem as informações e o devido controle gerencial, elas podem ter dificuldades de atuação no mercado.

Diante de tal problemática, Collins, Holzmann e Mendoza (1997) salientam que o orçamento desempenha um papel primordial para lidar com momentos de instabilidade, pois auxilia o gestor no processo de aferição entre as ameaças e as oportunidades. Becker et al. (2016) destacam que em um contexto de crises econômicas, as funções orçamentárias de planejamento, de alocação de recursos e de avaliação de desempenho podem sofrer modificações, comparativamente àquelas para as quais foram planejadas, em um contexto de economia estável. Por sua vez, o estudo de Bourmistrov e Kaarbøe (2017) aponta que a adoção de um controle orçamentário rigoroso foi uma resposta que decorreu das percepções dos gestores sobre a crise, bem como a demanda dos investidores e do conselho de administração, com o intuito de melhorar a situação financeira de curto prazo da organização. Ainda, indica que os gestores que observam mais as informações externas das organizações possuem mais chances de sobrevivência e de continuidade, do que aquelas que analisam apenas o ambiente interno (Bourmistrov \& Kaarbøe, 2017).

No entanto, Pavlatos e Kostakis (2018) ressaltam que a pouca atenção temse dado às pesquisas em contabilidade gerencial no que diz respeito à maneira como as organizações lidam com as crises econômicas envolvendo seu ambiente. Nesse mesmo sentido, complementam que as inovações em contabilidade gerencial geram informações de melhor qualidade e mais amplas para lidar com a incerteza gerada por crises econômicas (Pavlatos \& Kostakis, 2018). Diante do exposto, assume-se que os fatores que podem influenciar a modificação das funções do orçamento (em especial as funções de 
planejamento, de alocação de recursos e de avaliação de desempenho), devem ser observados, levando-se em consideração, também, aspectos endógenos, em especial a estratégia, o tamanho da organização e seu estágio de ciclo de vida.

Dessa maneira, o estudo centra atenção no seguinte problema de pesquisa: qual a relação entre as incertezas em um ambiente de crises econômicas e as variáveis endógenas com as funções do orçamento? Decorrente do problema de pesquisa tem-se como objetivo avaliar a relação entre as incertezas, em um ambiente de crises econômicas, e as variáveis endógenas com as funções do orçamento em indústrias catarinenses.

No contexto de pesquisas na área organizacional, as crises econômicas são apontadas por Ury e Smoke (1991) como uma ameaça substancial para as organizações. Já Kunc e Bhandari (2011) destacam como um momento de oportunidades e expansão no mercado. No entanto, autores como Hopwood (2009), Van der Stede (2011), Abernethy e Mundy (2014) relatam que as pesquisas em contabilidade gerencial têm dado pouca atenção acerca das respostas organizacionais para momentos de instabilidade econômica. Assim, a temática representa uma oportunidade de pesquisa em contabilidade gerencial e amplia o entendimento de como as ferramentas de controle gerencial, em especial, o uso do orçamento segregado em suas funções orçamentárias é remodelado para contribuir com o processo de enfrentamento de crises.

A contribuição prática deste estudo aponta para efeitos de aprendizagem e uma melhor percepção dos gestores para enfrentar possíveis crises econômicas no futuro e, de acordo com Messner (2016), que afirmam haver espaço para estudos em torno das práticas adotadas na gestão do setor industrial, em especial o orçamento.

Do exposto, o estudo contribui com a literatura; pois, conforme sugerido por autores como Burns e Scapens (2000), Hopwood (2009), Van der Stede (2011), Abernethy e Mundy (2014), centra atenção no SCG em um contexto de crises econômicas. Tal contribuição está por relacionar variáveis contingenciais (incerteza, ciclo de vida, tamanho, estratégia) como as modificam das funções orçamentárias um contexto de crises econômicas, comparativamente ao uso em um momento anterior à crise. O estudo, seguindo ao sugerido por Hopwood (2009), evidencia às orientações orçamentárias adotadas por indústrias frente às crises econômicas. A contribuição também decorre da confirmação, no contexto brasileiro, dos achados de Becker et al. (2016) para empresas europeias, no que se refere ao aumento de certas funções orçamentárias em detrimento de outras, em momentos de crises econômicas.

Também, contribui por avançar o estudo de Becker et al. (2016), uma vez que incorpora na análise o efeito das variáveis endógenas, indicando que o posicionamento estratégico amplia a intensidade do uso das funções orçamentárias; os estágios de iniciais do ciclo de vida elevam a importância da função orçamentária de avaliação de desempenho e que empresas maiores tendem a atribuir menos importância para essa função orçamentária. 


\section{REVISÃO DA LITERATURA}

\subsection{Crise econômica e o Uso das Funções do Orçamento}

A crise econômica é apontada por Sandroni (1994) como uma perturbação econômica, que deriva de escassez e de um desequilíbrio entre produção e consumo, consequentemente ocorre uma queda brusca na produção e na capacidade produtiva, levando à falência de empresas, ao aumento da taxa de desemprego, à diminuição de salários, bem como a diminuição do lucro e de preços, a restrição de investimentos, a alta liquidez bancária, dentre outros. Essas crises econômicas são consideradas como uma ameaça substancial para as organizações (Ury \& Smoke, 1991), uma vez que induzem a mudanças significativas no comportamento dos consumidores, altera a procura de bens e serviços, o que provoca variações na maneira como as organizações operam (Afthonidis \& Tsiotras, 2014). Além disso, as crises econômicas ao afetarem países e empresas trazem como consequências a indisponibilidade do capital e a redução na demanda, nesse contexto, segundo Becker et al. (2016), o uso das funções do orçamento pode desempenhar um papel essencial para lidar com tais crises, facilitando a adaptação organizacional na nova dinâmica do ambiente e contribui para ampliar a atenção dos gestores às ameaças e às oportunidades oriundas de um ambiente afetado por crises econômicas.

Ressalta-se que, em períodos de crises econômicas, muitas organizações são confrontadas com ameaças graves, que exigem ações imediatas, de curto prazo, visando assegurar a sobrevivência que, por vezes, também prejudicam a saúde financeira a longo prazo (Asel et al., 2011). Ademais, elas geralmente causam efeitos negativos sobre o desempenho das organizações e das atividades inovadoras (Brzozowski \& Cucculelli, 2016). Dessa maneira, Hopwood (2009) sugere que tais crises pressionam o funcionamento do SCG de modo contínuo, independente do ramo de atividade, influenciam a reformulação das funções do orçamentos, dada a possibilidades de modificação dos níveis de custos e receitas, dentre outros. Diante de tal fato, o autor indica a necessidade de reformulação constante do SCG frente às posturas estratégicas adotadas em períodos de recessão econômica.

Na mesma direção, Claessens, Djankov e Xu (2000) apontam que as crises econômicas e financeiras causam impactos no ambiente em que as organizações estão inseridas e afetam vários setores, alterando as condições de crédito, a demanda de produtos, os preços dos insumos, as relações entre as organizações e até mesmo a esfera política. Do mesmo modo, Collins, Almer e Mendoza (1999) relatam que crises econômicas, o ambiente e os aspectos governamentais afetam a postura estratégica de negócios nas organizações e repercutem no uso das funções do orçamento.

Por outro lado, as condições supracitadas não afetam todas as organizações com a mesma intensidade (Afthonidis \& Tsiotras, 2014). Para alguns, o ambiente é considerado hostil e concebe uma ameaça; para outras, pode ser visto como uma opção para explorar oportunidades e desenvolver-se no mercado (Kunc \& Bhandari, 2011). Nesse sentido, Afthonidis e Tsiotras (2014) sugerem que um fator essencial na determinação da postura estratégica adequada de uma organização que opera durante as crises econômicas, sendo fundamental compreender o impacto no ambiente micro e macroeconômico. 
Na mesma direção, para Brzozowski e Cucculelli (2016), os momentos de recessão gerados por crises econômicas podem ser vistos como uma oportunidade para aumentar a competividade, pois enquanto algumas empresas, em um mesmo setor de atividade, cortam gastos, as que conseguem "nadar contra a corrente" conseguem obter oportunidades e benefícios adicionais, sobretudo depois que a economia se recupera da crises econômicas (Brzozowski \& Cucculelli, 2016).

No ponto de vista de Waal e Mollema (2010), as organizações cujos gestores são sensíveis às características das circunstâncias em que operam, essas conseguem colocar em prática uma implementação de suas ações em um plano estratégico bem estruturado e formulado, consequentemente obtêm benefícios com crises econômicas. No entanto, para assegurar essas oportunidades, faz-se necessário também se certificar de um plano de investimentos para marketing ou anúncios, ou seja, áreas que colaboram para acrescentar valor à organização. Os autores, ainda, ressaltam que em períodos de resseção as organizações têm a oportunidade de inserir novos produtos no mercado, solidificar a sua posição, realizar fusões e aquisições e recrutar pessoal qualificado.

Em síntese, as crises econômicas podem representar uma ameaça para organizações que operam nesse ambiente, devido a superprodução e falta de demanda no mercado, a recessão econômica induz a redução do PIB e aumenta o desemprego. No contexto Brasileiro, dados do IBGE (2017) indicam que, no ano de 2015, o PIB recuou 3,8\% em relação a 2014 e em 2016 a queda foi de $3,6 \%$ (maiores quedas na série histórica iniciada em 1996). Por sua vez, de acordo com o IBGE (2017), nos anos de 2015 e 2016, o saldo entre admissões e demissões foi negativo, com uma redução no número de empregos formais de, aproximadamente, 1,54 milhão e 1,32 milhão, respectivamente. Por fim, as oscilações que acontecem em momentos de crises econômicas fazem com que ocorram a incapacidade de controlar fatores como a inflação, reduzir o volume de negociações e a rentabilidade das organizações, dentre outros. No entanto, embora a crise represente uma ameaça para algumas organizações, por outro lado oferece oportunidades, as quais variam com o ramo de atividade e capacidade gerencial.

Dentre as diversas lentes teóricas utilizadas para compreender o uso de ferramentas de controle gerencial, no estudo faz-se a opção pela teoria contingencial. Tal predileção está fundamentada em afirmativas como a de Otley (2016), ao relatar que no contexto das pesquisas em contabilidade gerencial, o papel da teoria contingencial é contribuir para o entendimento sobre as práticas de gestão utilizadas em circunstâncias específicas sobretudo, decorrente do fato de que não há universalidade do SGC.

Ademais, pautado em Beuren e Fiorentin (2014) e Otley (2016), a teoria contingencial procura compreender e explicar como variáveis contingenciais influenciam o crescimento e a sobrevivência das organizações. Dentre as diversas variáveis contingenciais observadas na literatura de controle gerencial, Chenhall (2003) destaca o ambiente (e a incerteza decorrente dele). Haja vista que o ambiente é uma variável exógena à organização e tem sido, de longa data, utilizada para ampliar a compreensão sobre a adoção de métodos de avaliação econômica e de gestão que afetam a maneira de gerenciar uma organização e seu desempenho (Hayes, 1977, Waterhouse \& Tiessen, 1978). Por sua vez, 
variáveis endógenas a exemplo do estágio do clico de vida, da estratégia e do tamanho são recorrentemente descritas como úteis para investigações baseadas na teoria contingencial (ver por exemplo Frezatti et al., 2010; Neitzke \& Espejo, 2016.)

Especificamente em relação ao impacto que a incerteza decorrente do ambiente de crises econômicas pode ocasionar no uso do orçamento, estudos como os de Campello, Graham e Harvey (2010) e Becker et al. (2016) sinalizam a importância de analisar as funções do orçamento (planejamento, alocação de recursos e avaliação de desempenho) em contextos de crise. Becker et al. (2016), ao focalizar a crise econômica europeia de 2008, constatou que a função orçamentária de planejamento aumenta em períodos de crises econômicas, uma vez que em tempos de crise os riscos de fracasso podem ser maiores, elevando a responsabilidade do gestor em não cometer erros na tomada de decisão e, por conseguinte, reduzir o impacto causado pela crise. Esses fatores elevam a atenção do planejamento orçamentário e da precisão na execução, o que aumenta a pressão sobre os gestores, para garantir o alcance dos objetivos organizacionais.

Por sua vez, segundo Campello et al. (2010) e Becker et al. (2016), em períodos de crises econômicas, a função orçamentária de alocação de recurso ganha relevância, em especial em decorrência da redução de receitas. Esse fator faz com que se reduza a possibilidade de financiamento com recursos oriundos das atividades organizacionais, bem como eleva a possibilidade de as organizações necessitarem da captação de recursos de terceiros, gerando um aumento da dívida, o que pode implicar problemas de liquidez. Sendo assim, Staw, Sandelands e Duton (1981) e Hopwood (2009) sugerem que assegurar os recursos oriundos das atividades organizacionais para manter a liquidez é uma questão essencial para as organizações ameaçadas por crises econômicas.

Outra função do orçamento, apontada no estudo de Becker et al. (2016), que foi afetada pelo contexto de crises econômicas é a avaliação de desempenho, na qual sugere a utilização do orçamento para a avaliação de subordinados e da unidade de negócios. Em períodos de crises econômicas, essa avaliação pode ser dificultosa devido aos objetivos orçamentais que se tornam difíceis de alcançar. Em razão disso, Haka e Krishnan (2005) e Becker et al. (2016) salientam que o elemento de incerteza de crises econômicas afeta a avaliação de desempenho com base em orçamentos. Decorrente do exposto, elaboraramse três hipóteses de pesquisa. A Tabela 1 contém a descrição das hipóteses e a indicação das relações esperadas. 


\section{Tabela 1}

Hipóteses da pesquisa

\begin{tabular}{l|c}
\hline Hipóteses & $\begin{array}{c}\text { Relação esperada } \\
\text { Relação positiva } \\
(+)\end{array}$ \\
Hunção orçamentária de planejamento. & $\begin{array}{c}\text { Relação positiva } \\
(+)\end{array}$ \\
\hline $\begin{array}{l}\mathrm{H}_{2} \text { : Quanto maior a percepção de crise econômica, maior é o uso da } \\
\text { função orçamentária de alocação de recursos. }\end{array}$ & $\begin{array}{c}\text { Re } \\
\mathrm{H}_{3:} \text { Quanto maior a percepção de crise econômica, menor é o uso } \\
\text { da função orçamentária de avaliação de desempenho. }\end{array}$ \\
$\begin{array}{c}\text { Relação negativa } \\
(-)\end{array}$ \\
\hline
\end{tabular}

Fonte: Elaborado pelos autores.

\section{PROCEDIMENTOS METODOLÓGICOS}

Para o cumprimento do objetivo proposto esta pesquisa adota uma abordagem de pesquisa quantitativa e quanto aos procedimentos configura-se como levantamento. O período de coleta de dados ocorreu entre os meses de maio de 2017 a junho de 2017. A população é composta por dois mil gestores de indústrias catarinenses com perfil ativo na rede de relacionamento Linkedln ${ }^{\circledR}$, os quais a partir do filtro de busca aceitaram conexão com um dos autores da pesquisa. Para o filtro de busca, empregaram-se os termos: 'Santa Catarina' 'Indústria'. Acompanhadas pelos perfis dos respondentes como: 'Gestor' 'Diretor administrativo' 'Diretor financeiro' 'Gerente' 'Contador' 'Controller' 'Coordenador' 'Presidente' e 'Supervisor'. O processo resultou em 1.300 conexões, desse total, obtiveram-se 258 questionários válidos. A Tabela 2 apresenta a amostra da pesquisa.

\section{Tabela 2}

Amostra da pesquisa

\begin{tabular}{l|c}
\hline Item & Total \\
\hline Total de respondentes & 296 \\
\hline Indústrias que utilizam o orçamento & 260 \\
\hline Indústrias que declararam não utilizam o orçamento & 36 \\
\hline Exclusões decorrente de repostas inválidas & 2 \\
\hline Amostra final & 258 \\
\hline
\end{tabular}

Fonte: Dados da pesquisa.

A escolha das indústrias do Estado de Santa Catarina como amostra justificase por ser o quarto Estado em quantidade de empresas, o quinto em quantidade de trabalhadores e, ainda, o segundo Estado com maior participação da indústria de transformação no Produto Interno Bruto (PIB). Ademais, a economia catarinense destaca-se pela diversificação de seu polo industrial (FIESC, 2017), sendo relevante observar que, segundo o Instituto Brasileiro de Geografia e Estatística (IBGE, 2017), o estado de Santa Catarina sofreu os efeitos da crise econômica brasileira de forma mais aguda em relação à média nacional. Dados indicam que no ano de 2016, enquanto o Estado catarinense sofreu uma retração de seu PIB na ordem de $4,2 \%$, a média nacional foi uma diminuição de 3,6\%. 0 referido estudo indica também que a crise econômica atingiu de forma mais 
intensa o setor industrial catarinense, uma vez que ele representa $28,7 \%$ do PIB catarinense e $22,5 \%$ no país.

Na Tabela 3, é exposto o cargo ocupado pelos respondentes, bem como a proporção em relação à amostra total.

\section{Tabela 3}

Respondentes da pesquisa, em ordem alfabética de cargo

\begin{tabular}{l|c|c}
\hline Cargo & $\mathbf{n}_{\mathbf{i}}$ & $\mathbf{f}_{\mathbf{i}}$ \\
\hline Analista & 16 & 6,20 \\
Financeiro/Orçamento & 10 & 3,88 \\
\hline Contador & 12 & 4,65 \\
\hline Controller & 34 & 13,18 \\
\hline Coordenador & 25 & 9,69 \\
\hline Diretor administrativo & 15 & 5,81 \\
\hline Diretor financeiro & 85 & 32,95 \\
\hline Gerente & 8 & 3,10 \\
\hline Outros* & 24 & 9,30 \\
\hline Presidente & 14 & 6,59 \\
\hline Sócio Gerente/Proprietário & 12 & 4,65 \\
\hline Supervisor & $\mathbf{2 5 8}$ & $\mathbf{1 0 0 , 0 0}$ \\
\hline Total &
\end{tabular}

Nota: $n_{i}$ frequência acumulada; fi rrequência relativa.

* Outros contempla respondentes cujo cargo declarado foram: Administrador $(0,4 \%)$, Financeiro (0,4\%), Engenheiro (0,8\%), Diretor Industrial/Operações (1,6\%).

Fonte: Dados da pesquisa.

As maiores quantidades de respondentes referem-se a 85 gerentes industriais, representando $32,95 \%$ da amostra. Ainda são apontados 34 coordenadores, 25 diretores administrativos, 24 presidentes, 15 diretores financeiros, 12 controllers, 12 supervisores, 10 contadores, os demais respondentes correspondem às outras categorias de funções dentro das organizações. Na sequência, a Tabela 4 expõe a amostra da pesquisa segregada pela classificação do Código Nacional de Atividades Econômicas (CNAE) das indústrias de transformação, conforme IBGE (2017). 


\section{Tabela 4}

Classificação das indústrias, segundo CNAE, em ordem de participação

\begin{tabular}{|c|c|c|c|}
\hline Categoria & $\mathbf{n}_{\mathbf{i}}$ & $f_{i}$ & $\mathbf{F}_{\mathbf{i}}$ \\
\hline 1. Fabricação de produtos têxteis & 36 & 13,95 & 13,95 \\
\hline 2. Fabricação de produtos de borracha e de material plástico & 32 & 12,40 & 26,35 \\
\hline 3. $\quad$ Fabricação de produtos diversos & 22 & 8,53 & 34,88 \\
\hline 4. Metalurgia & 21 & 8,14 & 43,02 \\
\hline 5. Fabricação de máquinas e equipamentos & 20 & 7,75 & 50,77 \\
\hline 6. Confecção de artigos do vestuário e acessórios & 18 & 6,98 & 57,75 \\
\hline $\begin{array}{l}\text { 7. Manutenção, reparação e instalação de máquinas e } \\
\text { equipamentos }\end{array}$ & 18 & 6,98 & 64,73 \\
\hline 8. $\quad$ Fabricação de produtos alimentícios & 17 & 6,59 & 71,32 \\
\hline 9. $\quad$ Fabricação de celulose, papel e produtos de papel & 9 & 3,49 & 74,81 \\
\hline 10. Fabricação de produtos de madeira & 9 & 3,49 & 78,30 \\
\hline $\begin{array}{l}\text { 11. Fabricação de produtos de metal, exceto máquinas e } \\
\text { equipamentos }\end{array}$ & 9 & 3,49 & 81,79 \\
\hline 12. Fabricação de móveis & 8 & 3,10 & 84,89 \\
\hline $\begin{array}{l}\text { 13. Fabricação de equipamentos de informática, produtos } \\
\text { eletrônicos e ópticos }\end{array}$ & 7 & 2,69 & 87,58 \\
\hline 14. Fabricação de máquinas, aparelhos e materiais elétricos & 5 & 1,94 & 89,52 \\
\hline 15. Fabricação de produtos químicos & 5 & 1,94 & 91,46 \\
\hline Outros grupos (9 grupos distintos) & 22 & 8,50 & 100 \\
\hline Total & 258 & 100 & \\
\hline
\end{tabular}

Nota: $f_{i}$ frequência relativa; $F_{i}$ Frequência absoluta; $n_{i}$ frequência acumulada

Fonte: Dados da pesquisa.

A segregação da amostra segue a classificação das indústrias de transformação que, de acordo com os critérios estabelecidos pelo IBGE, contempla 24 divisões. Constata-se que o segmento predominante do estudo é o de fabricação de produtos têxteis $(13,95 \%$ da amostra) precedido do setor de fabricação de produtos de borracha e de material plástico, representou $12,40 \%$ da amostra, com participação de 32 respondentes.

Para a coleta de dados, foi utilizado um questionário adaptado da pesquisa de Becker et al. (2016), cuja medição foi por escala intervalar de 0 a 10 pontos. Realizou-se a tradução e a revisão do questionário do inglês para o português por um profissional especialista em língua inglesa e, posteriormente, aplicou-se um pré-teste com professores doutores, doutorandos e profissionais com conhecimento na área do estudo e possuidores de características semelhantes à população do estudo. Cabe ressaltar que os respondentes e os questionários aplicados no pré-teste não foram incluídos na amostra da pesquisa.

Os dados foram coletados com auxílio do programa Google Docs online e enviados por meio de link eletrônico. O questionário foi encaminhado via Linkedln $\AA$ para os gestores previamente contatados.

As variáveis empregadas nos procedimentos de análise são sintetizadas na Tabela 5, a qual contempla a categoria de análise, as questões correspondentes a cada variável, bem como os autores que já as utilizaram em estudos correlatos. 
Tabela 5

Constructo da pesquisa

\begin{tabular}{|c|c|c|c|}
\hline Variável & Categoria & Questões & Autores \\
\hline $\begin{array}{l}\text { Ambiente de } \\
\text { crise (AC) }\end{array}$ & $\begin{array}{l}\text { Percepção da crise } \\
\text { econômica }\end{array}$ & 1 a 7 & $\begin{array}{c}\text { Gordon e Narayanan } \\
\text { (1984); Janke, Mahlendorf } \\
\text { e Weber (2014); Becker et } \\
\text { al. (2016). }\end{array}$ \\
\hline \multirow{3}{*}{$\begin{array}{l}\text { Incerteza } \\
\text { ambiental } \\
\text { (PEU) }\end{array}$} & Intensidade de concorrência & \multirow{3}{*}{20 a 31} & \multirow{3}{*}{$\begin{array}{c}\text { Gordon e Narayanan } \\
\text { (1984); Janke et al. (2014); } \\
\text { Becker et al. (2016). }\end{array}$} \\
\hline & Imprevisibilidade do ambiente & & \\
\hline & $\begin{array}{c}\text { Elementos de mudança } \\
\text { ambiental }\end{array}$ & & \\
\hline \multirow{3}{*}{$\begin{array}{l}\text { Funções do } \\
\text { orçamento } \\
\text { (FO) }\end{array}$} & Planeiamento & 8 a 11 e 16; & \multirow{3}{*}{ Becker et al. (2016). } \\
\hline & Alocação de recursos & $\frac{17}{12 \mathrm{e} 13}$ & \\
\hline & Avaliação de desempenho & $14 ; 15 ; 18 ; 19$. & \\
\hline \multirow{2}{*}{$\begin{array}{l}\text { Estratégia } \\
\text { (EST) }\end{array}$} & Custo & \multirow{2}{*}{32 a 53} & \multirow{2}{*}{$\begin{array}{l}\text { Porter (1980); Janke et al. } \\
\text { (2014); Becker et al. } \\
\text { (2016). }\end{array}$} \\
\hline & Diferenciação & & \\
\hline \multirow{5}{*}{$\begin{array}{l}\text { Ciclo de vida } \\
\text { (CV) }\end{array}$} & Nascimento & \multirow{5}{*}{54} & \multirow{5}{*}{$\begin{array}{c}\text { Miller e Friesen (1986); } \\
\text { Davila e Foster (2007); } \\
\text { Moores e Yuen (2001); } \\
\text { Janke et al. (2014); } \\
\text { Becker et al. (2016). }\end{array}$} \\
\hline & Crescimento & & \\
\hline & Maturidade & & \\
\hline & Renascimento & & \\
\hline & Declínio & & \\
\hline $\begin{array}{l}\text { Tamanho } \\
\text { (TAM) }\end{array}$ & Receita Operacional Bruta & Painel B & $\begin{array}{c}\text { Janke et al. (2014); } \\
\text { Becker et al. (2016); } \\
\text { BNDES (2017). }\end{array}$ \\
\hline
\end{tabular}

Fonte: Dados da pesquisa.

Para mensuração da variável Ambiente de Crise, o questionário contemplou sete questões, no qual o respondente sinalizou sua percepção sobre o ambiente de crise econômica. Como se trata de uma escala de percepção individual, mesmo que os respondentes estejam na mesma unidade da federação, possuem percepções diferentes em parte decorrentes do tipo de produto da empresa, relação com clientes, mercado de atuação, dentre outros.

No questionário, as questões 8 a 19 (Funções Orçamentárias) previam dupla resposta - no primeiro momento (grupo 0) os respondentes reportavam a percepção sobre o uso das funções orçamentárias de planejamento, alocação de recursos e avaliação de desempenho no período economicamente estável (anterior à crise econômica) para, na sequência, posicionarem-se como utilizam as mesmas funções no contexto de crise econômica de 2016 (grupo 1).

Os dados coletados foram tabulados em planilhas eletrônicas de excel. $\mathrm{Na}$

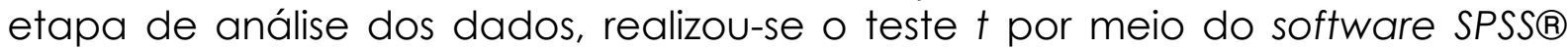
Statistical versão 22, com as funções orçamentárias visando testar se as médias são iguais ou não para o período anterior e o durante a crise. Fávero, Belfiore, Silva e Chan (2009) apontam que o teste t é aplicado quando visa verificar se as médias de duas amostras relacionadas $\left(X_{1}, \ldots, Y_{n}, Y_{1}, \ldots, Y_{n}\right)$ com distribuição normal, sendo estas extraídas da mesma população são ou não significativamente diferentes. Posteriormente, realizou-se o teste de correlação canônica, visando analisar a relação de incertezas em um ambiente de crises econômicas e variáveis endógenas com a função orçamentária de planejamento (a), alocação de recursos (b) e avaliação de desempenho (c) em 
indústrias catarinenses, no período durante a crise econômica, comparativamente ao período anterior.

A correlação canônica pode ser vista como uma extensão da regressão múltipla. Por meio dela, pode-se avaliar as inter-relações entre dois conjuntos de variáveis, compostas por combinações lineares dos vários caráteres que os constituem, conforme apontam Hair Jr., Anderson, Tatham e Black (2005). Na sequência, apresentam-se os resultados da pesquisa que além de expor os principais achados e a análise, descreve aspectos pontuais do processo de investigação.

\section{ANÁLISE DOS RESULTADOS}

Inicia-se a análise pela identificação da influência do ambiente de crise econômica de 2016 para as funções orçamentárias em indústrias catarinenses obtidos a partir do teste $t$. Por meio desse teste, foi possível identificar as médias atribuídas pelos gestores para cada uma das três funções orçamentárias investigadas antes e durante o período de crise econômica, bem como se tais mudanças foram significativas. A Tabela 6 sumariza os resultados.

\section{Tabela 6}

Teste de médias das funções orçamentárias

\begin{tabular}{|c|c|c|c|c|c|}
\hline Dados & Grupo* & Qtd & Média & $\mathbf{T}$ & Sig. \\
\hline \multirow{2}{*}{ Planejamento } & 0 & 258 & 48,43 & \multirow{2}{*}{22409,500} & \multirow{2}{*}{0,000} \\
\hline & 1 & 258 & 54,17 & & \\
\hline \multirow{2}{*}{ Alocação de Recursos } & 0 & 258 & 15,62 & \multirow{2}{*}{21659,000} & \multirow{2}{*}{0,000} \\
\hline & 1 & 258 & 17,86 & & \\
\hline \multirow{2}{*}{$\begin{array}{l}\text { Avaliação de } \\
\text { Desempenho }\end{array}$} & 0 & 258 & 32,64 & \multirow{2}{*}{7763,000} & \multirow{2}{*}{0,000} \\
\hline & 1 & 258 & 29,66 & & \\
\hline
\end{tabular}

Nota: 0 para antes da crise; 1 para durante a crise.

Observação: Grupo 0 - antes do período de crise; Grupo 1 - durante o período de crise econômica.

Fonte: Dados da pesquisa.

No Grupo 0, os respondentes sinalizaram como empregaram o orçamento em momentos estáveis e no Grupo 1, os mesmos respondentes sinalizaram como estavam utilizando o orçamento no momento de crise econômica de 2016. Percebe-se que houve variação estatisticamente significativa no valor das médias referente aos períodos antes (Grupo 0) e durante a crise econômica (Grupo 1) nas três funções orçamentárias, todos com nível de significância de $1 \%$. Decorrente dos resultados, é possível inferir que a utilização do orçamento para o planejamento, nas indústrias catarinenses, foi modificada durante a crise econômica de 2016.

Ao comparar as médias obtidas, os resultados indicam que, no período de crise econômica, os gestores das indústrias catarinenses elevaram a importância atribuída para a função orçamentária de planejamento. Para a função orçamentária de alocação de recursos, os resultados indicam que os gestores atribuem uma importância maior para a utilização do orçamento como uma ferramenta de apoio à alocação dos recursos no período de crise observado. Resultado condizente com a necessidade de uma melhor alocação de recursos 
os quais, decorrente do ambiente de crises econômicas, podem se tornar escassos por possibilitar elevar o controle sobre o poder de decisão para a realização de despesas e autorização de gastos.

Por sua vez, para a função orçamentária de avaliação de desempenho, no período anterior e durante a crise econômica, constata-se haver uma redução na declaração de sua utilização e tais resultados sinalizam haver diminuição de sua importância em tempos de crise. Pode-se inferir que, em tempos de crise econômica, as indústrias observadas consideram ser de menor relevância a utilização da função orçamentária de avaliação de desempenho para estabelecer a compensação de gestores, visto que atingir as metas orçamentárias se torna complexo devido às oscilações do mercado. Sumariamente, os resultados indicam que a utilização das funções orçamentárias nas empresas da amostra é afetada pelo ambiente da crise econômica de 2016, que se observou pela modificação das funções do orçamento nas organizações. No estudo Becker et al. (2016), constatou-se que tais achados estão alinhados à crise econômica no contexto de empresas europeias.

Quanto aos resultados da análise de correlação canônica, o modelo avalia a relação de incertezas em um ambiente de crise econômica e variáveis endógenas com as funções do orçamento. Primeiramente, apresenta-se a matriz de correlação entre todas as variáveis do estudo, proporcionando a visualização das possíveis associações entre as variáveis utilizadas nos modelos da correlação, conforme o exposto na Tabela 7.

\section{Tabela 7}

Correlação Canônica das funções orçamentárias com as variáveis contingenciais

\begin{tabular}{c|c|c|c|c|r|r}
\hline Número & AutoValor & $\begin{array}{c}\text { Correlação } \\
\text { Canônica }\end{array}$ & Wilk Lambda & Chi-Square & D.F. & P-Value \\
\hline 1 & 0,12658700 & 0,3557900 & 0,832108 & 46,40770 & 15 & $\mathbf{0 , 0 0 0 0 ^ { * }}$ \\
\hline 2 & 0,04519120 & 0,2125820 & 0,952708 & 12,23270 & 8 & 0,1411 \\
\hline 3 & 0,00219987 & 0,0469028 & 0,997800 & 0,55608 & 3 & 0,9064 \\
\hline
\end{tabular}

Fonte: Dados da pesquisa.

Verifica-se que foram calculadas três combinações lineares diferentes do conjunto de variáveis apresentadas na tabela 8. A primeira apresentou-se significativa quanto ao nível de $5 \%$, com p-Value de 0,0000. O coeficiente de correlação canônica de 0,3557 desta combinação linear, segundo Hair Jr. et al. (2005) concebe uma força de associação moderada entre as variáveis do modelo, obtendo um poder de explicação de 35,57\% do modelo. Na Tabela 8 , visualizam-se os coeficientes para as variáveis canônicas do grupo de variáveis contingenciais e do grupo que representa as três funções orçamentárias deste estudo. 
Tabela 8

Correlação canônica

\begin{tabular}{c|c|c}
\hline Grupos & Variáveis & Coeficientes \\
\hline \multirow{4}{*}{$\begin{array}{c}\text { Variáveis contingenciais } \\
\text { (VC) }\end{array}$} & Tamanho (TAM) & $-0,116508$ \\
\cline { 2 - 3 } & Ambiente de Crise (AC) & $-0,017931$ \\
\cline { 2 - 3 } & Incerteza Ambiental Percebida (PEU) & $-0,646969$ \\
\cline { 2 - 3 } & Ciclo de Vida (CV) & $-0,173107$ \\
\hline \multirow{2}{*}{$\begin{array}{c}\text { Funções Orçamentárias } \\
\text { (FO) }\end{array}$} & Estratégia (EST) & $-0,598881$ \\
\cline { 2 - 3 } & Planejamento (PLA) & $-0,474223$ \\
\cline { 2 - 3 } & Alocação de Recursos (AR) & $-0,424084$ \\
\hline
\end{tabular}

Fonte: Dados da pesquisa.

Foram analisados os coeficientes apresentados na primeira coluna da Tabela 7, em decorrência de que essa representa maior correlação entre as três combinações lineares das funções orçamentárias, conforme demonstrado na Tabela 8. Por meio software Statgraphics ${ }^{\circledR}$, os coeficientes da primeira equação canônica foram descritos entre as variáveis contingenciais e a segunda equação os valores das funções orçamentárias:

\section{$V C=-0,116508 * T A M--0,017931 * A C-0,646969 * P E U-0,173107 * C V-$ $0,598881 *$ EST, e FO $=-0,474223 *$ PLA $-0,424084^{*} A R+1,41046 * A D$}

Assim, ao relacionar as funções orçamentárias com as incertezas em um ambiente de crise econômica e variáveis endógenas, no período durante a crise econômica de 2016, comparativamente ao período antes da crise econômica, percebe-se que há uma relação diretamente proporcional entre as variáveis de planejamento e de alocação de recursos e as variáveis contingenciais de tamanho, ambiente de crise, incerteza ambiental percebida, ciclo de vida e estratégia. No entanto, para a variável avaliação de desempenho a relação com as demais variáveis analisadas foi inversamente proporcional.

Por conseguinte, para as funções orçamentárias de planejamento e de alocação de recursos, que são diretamente proporcionais às variáveis contingentes, leva-se em consideração a força da relação estabelecida entre as variáveis, uma vez que é possível constatar, na seguinte ordem de relevância que i) a percepção dos gestores das indústrias catarinenses, em relação ao nível de incerteza do ambiente (PEU), é diretamente proporcional à intensidade do uso das funções orçamentárias de planejamento e alocação de recursos; ii) quanto mais tendente para um posicionamento estratégico (EST) de diferenciação, maior será a intensidade do uso das funções orçamentárias de planejamento e alocação de recursos; iii) quanto maior o tamanho (TAM) das indústrias catarinenses, mensurado pelo faturamento, maior será a intensidade do uso das funções orçamentárias de planejamento e alocação de recursos; iv) quanto mais avançado for o estágio do ciclo de vida (CV) da organização, maior será o uso das funções orçamentárias de planejamento e alocação de recursos; v) com menor força de relação entre as variáveis analisadas, constata-se que quanto maior for a percepção do ambiente de crise (AC) econômica, maior será o uso das funções orçamentárias de planejamento e alocação de recursos das empresas. 
Por sua vez, para a função orçamentária de avaliação de desempenho, constata-se que no modelo é inversamente proporcional às variáveis contingentes, levando-se em consideração a força da relação estabelecida entre as variáveis, é possível estabelecer, na seguinte ordem de relevância que: i) quanto maior a percepção dos gestores das indústrias catarinenses, em relação ao nível de incerteza do ambiente (PEU), menor é a intensidade do uso da função orçamentária de avaliação de desempenho; ii) quanto mais a indústria catarinense tender para um posicionamento estratégico (EST) de liderança em custo maior é a intensidade do uso da função orçamentária de avaliação de desempenho; iii) quanto maior o tamanho (TAM) das indústrias catarinenses, mensurado pelo faturamento, menor é a intensidade do uso da função orçamentária de avaliação de desempenho; iv) empresas em estágio de nascimento e crescimento (CV) tendem a utilizar com mais intensidade a função orçamentária de avaliação de desempenho, comparativamente aquelas em estágios de maturidade, renascimento e declínio; v) quanto maior for a incerteza em relação à crise econômica $(\mathrm{AC})$ menor será o uso da função orçamentária de avaliação de desempenho.

Tais achados permitem não rejeitar as três hipóteses de pesquisa, ou seja, $\mathrm{H}_{1}$ - Quanto maior a percepção de crise econômica, maior é o uso da função orçamentária de planejamento (relação positiva); $\mathrm{H}_{2}$ - Quanto maior a percepção de crise econômica, maior é o uso da função orçamentária de alocação de recursos (relação positiva); $\mathrm{H}_{3}$ - Quanto maior a percepção de crise econômica, menor é o uso da função orçamentária de avaliação de desempenho (relação negativa). Estes achados contrariam os estabelecidos por Pavlatos e Kostakis (2018) os quais, ao investigarem as práticas de contabilidade gerencial durante o período de crise econômica na Grécia, concluem que no período de crise econômica os gestores indicaram confiarem no desenvolvimento de orçamentos anuais para o controle de custos, bem como na avaliação de desempenho e planejamento, priorizando soluções e planos de ações de longo prazo.

Ao avaliar as três funções orçamentárias consta-se que os resultados estão em consonância com Billings, Milburn e Schaalman (1980) que indicam que, nas situações novas e incertas, os gestores possuem uma compreensão mais limitada do ambiente em que estão inseridas. Além disso, os estudos de Janke et al. (2014) apontaram a crise econômica como uma das variáveis ambientais de maior impacto quanto às incertezas do ambiente organizacional, em consonância com Gordon e Narayanan (1984) os quais indicaram que as organizações precisam de informações mais detalhadas e completas quando se encontram em um ambiente de alta incerteza.

Nesse contexto, o uso da função orçamentária de planejamento possivelmente contribui para reduzir as incertezas. Palermo e Van Der Stede (2011) corroboram afirmando que os planejamentos nas organizações devem ser reavaliados e atualizados em conformidade com o ambiente em que se estão inseridos, para uma melhor tomada de decisão e obtenção de resultados positivos. Na visão de Becker et al. (2016), identificaram em seu estudo que o planejamento orçamentário diminui as incertezas relativas ao processo de tomada de decisão e, ainda em um contexto de crise econômica, faz com que os gestores observem de forma mais detalhada o ambiente organizacional com 
o intuito de prever o impacto da crise, bem como os efeitos em relação às vendas, à produção, às despesas, ao lucros e à liquidez.

O mesmo ocorre quanto à função orçamentária de alocação de recursos. O uso dessa função é aumentado ou diminuído conforme o aumento ou a diminuição da percepção do ambiente de crise e as incertezas desses ambientes. Isso também é observado nas demais variáveis de tamanho, estratégia e ciclo de vida. Pode-se concluir, portanto, que a percepção de crise, incerteza ambiental percebida, bem como variáveis endógenas (tamanho, ciclo de vida e estratégia) possuem influência no uso da função orçamentária de alocação de recursos. Esses resultados corroboram com Hopwood (2009) que destacou a necessidade de assegurar o uso produtivo dos recursos, além da gestão de liquidez, como uma ação necessária para as organizações ameaçadas pela crise. Ainda, de acordo com Zona (2012), nos ambientes incertos de crises econômicas a conservação dos recursos é requerida, assim os gestores precisam centralizar as decisões em torno da maneira mais coerente para a alocação ou realocação de recursos, bem como o gasto com despesas.

Por outro lado, a relação entre a função orçamentária de avaliação de desempenho e as variáveis contingenciais de ambiente de crise, incerteza ambiental percebida, variáveis endógenas (tamanho, ciclo de vida e estratégia) não foi estatisticamente significativa para o contexto analisado, decorrente disso, não é possível inferir sobre tais relações. Embora, estudos anteriores (Haka \& Krishnan, 2005; Becker et al., 2016) indiquem que ambientes incertos e de crises econômicas geram mudanças constantes, as quais dificultam o ápice de metas e objetivos propostos no orçamento e na avaliação do desempenho do gestor ou da organização. Em consonância, os autores supracitados aduzem que em contextos de elevada incerteza em relação ao ambiente a medição do desempenho ou avaliação dos gestores/ unidade de negócios pode ser de forma não precisa, ou seja, o uso da função orçamentária de avaliação de desempenho seria inviável.

\section{CONCLUSÃO}

O objetivo da pesquisa foi avaliar a relação de incertezas em um ambiente de crise econômica e variáveis endógenas com as funções do orçamento em indústrias catarinenses. Caracterizou-se como quantitativa e tem como teoria de base a teoria contingencial. Como resultado, constatou-se que a incerteza causada pela crise econômica influencia a modificação do uso das funções orçamentárias de planejamento, alocação de recursos e avaliação de desempenho nas indústrias catarinenses.

Considerando que o ambiente de crises econômicas amplia o nível de incerteza no processo decisório, os gestores devem elevar a atenção para a continuidade das organizações e para o alcance dos resultados. Nesse contexto, contatou-se que a função de planejamento, auxilia na diminuição do nível de incerteza em torno da tomada de decisão, favorecendo a previsão mais assertiva do impacto e dos efeitos causados pelas crises econômicas, visando a ações futuras de curto, médio e longo prazo.

Do mesmo modo, a função orçamentária de alocação de recursos contribui, em momentos de crise econômica, auxiliar os gestores a ampliarem o 
controle e a tomada de decisão relacionadas com recursos e gastos, prevenindo a ineficiência e ampliando o poder de decisão e de autorização dos gastos, dispondo-se a uma melhor gestão dos recursos disponíveis na organização. Dessa maneira, os gestores podem melhorar a gestão de liquidez de curto e médio. Observou-se também, na pesquisa, que a função orçamentária de avaliação de desempenho tende a ser utilizada com mais cautela e em menor grau, nos períodos de oscilações causadas por crises econômicas, dada a dificuldade apontada para realizar a medição de desempenho do gestor e da própria empresa.

Os achados apontam que, no contexto investigado, a utilização das funções orçamentárias de planejamento e de alocação de recursos sofrem influência decorrente do tamanho da indústria, de seu estágio de ciclo de vida e da postura estratégica utilizada (diferenciação), o que não ocorre na função de avaliação de desempenho. Ademais, quando seus gestores possuem uma maior percepção de incerteza em relação ao ambiente de maneira geral e, especificamente, quanto ao ambiente de crises econômicas.

Conclui-se que a percepção dos gestores das indústrias catarinenses, em relação ao nível de incerteza do ambiente, é diretamente proporcional à intensidade do uso das funções orçamentárias de planejamento e de alocação de recursos. Observa-se, ainda, que quanto maior a tendência para um posicionamento estratégico de diferenciação, maior será a intensidade do uso das funções orçamentárias de planejamento e alocação de recursos. Esse resultado contraria a literatura, em especial aos estudos sobre beyond budgeting por duas razões. Em primeiro lugar pelo número expressivo de empresas que declararam utilizar o orçamento, comparativamente aquelas que não utilizam, indicando que o orçamento, nas indústrias catarinenses, é uma ferramenta de gestão amplamente utilizada. Em segundo lugar por indicar que, para o contexto analisado, as funções orçamentárias de planejamento e alocação de recursos têm sido amplamente empregadas por empresas.

Por sua vez, no que diz respeito as funções do orçamento para avaliação de desempenho, constata-se que, para o contexto em análise, o posicionamento estratégico de liderança em custo intensifica o uso dessa função. Nos estágios iniciais do ciclo de vida (nascimento e crescimento), as indústrias catarinenses tendem também, a elevar a importância da função orçamentária de avaliação de desempenho, comparativamente àquelas em estágios de maturidade, renascimento e declínio. Em um oposto, empresas maiores dão menos importância para essa função orçamentária. Constata-se também que a elevação da percepção dos gestores quanto às incertezas provenientes do ambiente e, especificamente, aquelas decorrentes do contexto de crises econômicas, reduzem a intensidade de uso da função orçamentária de avaliação de desempenho.

Conclui-se que a utilização das funções orçamentárias, nas indústrias catarinenses, é afetada pelo ambiente de crises econômicas, ou seja, há uma modificação no uso das funções do orçamento nas organizações. No período de crise econômica, os gestores das indústrias catarinenses elevaram a importância atribuída para as funções orçamentárias de planejamento e de alocação dos recursos. Em um oposto, decorrente do aumento da instabilidade do ambiente, a função orçamentária de avaliação de desempenho é diminuída em períodos 
de crise econômica, tanto para mensurar o desempenho dos gestores quanto o da empresa.

Além dos resultados expostos, evidencia-se a relevância teórica de tais achados bem como a contribuição para com a área de pesquisa de contabilidade gerencial. Essa trouxe respostas à literatura e ampliou o entendimento do uso das funções orçamentárias que podem ser remodeladas visando contribuir com a gestão organizacional no processo de enfrentamento de crises econômicas, no qual se verificaram atributos tanto no ambiente interno quanto no externo. Em uma perspectiva prática, os achados contribuem por indicar aos gestores que as funções orçamentárias devem ser utilizadas em consonância com o contexto. Em especial que as funções de planejamento e alocação de recursos devem ser sensíveis a fatores endógenos, como no caso do estágio de evolução (ciclo de vida) e a estratégia adotada, bem como as indústrias tendem a reduzir a importância das funções do orçamento para avaliação de desempenho, sugerindo atenção dos gestores para os efeitos positivos e negativos de seu uso, tanto em momentos de crise, quanto durante o processo de expansão/crescimento.

Desse modo, o estudo contribui apresentando dados para a formação dos primeiros pareceres sobre as funções do orçamento em indústrias catarinenses em ambientes de crises econômicas. Como limitação de pesquisa, destaca-se a percepção dos respondentes sobre as questões observadas e a transversalidade do estudo. Nesse sentido, estudos longitudinais, podem iniciar e acompanhar as funções orçamento no contexto de crise econômica e, determinar possíveis modificações no período pós-crise. Estudos de casos podem ampliar o entendimento sobre como tais modificações ocorrem em contextos específicos, bem como o estudo pode ser estendido para outros setores econômicos e contextos geográficos.

\section{REFERÊNCIAS}

Abernethy, M., \& Mundy, J. (2014). Uncertainty as a determinant of performance measurement and compensation systems: A review of the literature. In Management control and uncertainty (pp. 114-133). Palgrave Macmillan, London.

Afthonidis, E. P., \& Tsiotras, G. D. (2014). Strategies for business excellence under an economic crisis. The TQM Journal, 26(6), 610-624. https://doi.org/10.1 108/TQM10-2012-0078

Asel, J. A., Posch, A., \& Speckbacher, G. (2011). Squeezing or cuddling? The impact of economic crises on management control and stakeholder management. Review of Managerial Science, 5(2-3), 213-231. DOI $10.1007 / \mathrm{s} 11846-010-0051-4$

Becker, S. D., Mahlendorf, M. D., Schäffer, U., \& Thaten, M. (2016). Budgeting in times of economic crisis. Contemporary Accounting Research, 33(4), 14891517. https://doi.org/10.1111/1911-3846.12222 
Beuren, I. M., \& Fiorentin, M. (2014). Influência de Fatores Contingenciais nos Atributos do Sistema de Contabilidade Gerencial: um estudo em empresas têxteis do Estado do Rio Grande do Sul. Revista de Ciências da Administração, 16(38), 196-212. http://dx.doi.org/10.5007/21758077.2014v16n38p196

Billings, R. S., Milburn, T. W., \& Schaalman, M. L. (1980). A model of crisis perception: A theoretical and empirical analysis. Administrative Science Quarterly, 300316. https://doi.org/10.2307/2392456

BNDES. Banco Nacional de Desenvolvimento Econômico e Social. (2017). Disponível em: https://www.bndes.gov.br/wps/portal/site/home/financiamento/guia/quempode-ser-cliente/. Acesso em: 07 de julho de 2017.

Bourmistrov, A., \& Kaarbøe, K. (2017). Tensions in managerial attention in a company in crisis. Journal of Accounting \& Organizational Change, 13(2), 239261. https://doi.org/10.1108/JAOC-08-2015-0066

Brzozowski, J., \& Cucculelli, M. (2016). Proactive and reactive attitude to crisis: evidence from European firms. Entrepreneurial Business and Economics Review, 4(1), 181-191.

Burns, J., \& Scapens, R. W. (2000). Conceptualizing management accounting change: an institutional framework. Management accounting research, 11 (1), 3-25. https://doi.org/10.1006/mare.1999.0119

Campello, M., Graham, J. R., \& Harvey, C. R. (2010). The real effects of financial constraints: Evidence from a financial crisis. Journal of financial Economics, 97(3), 470-487. https://doi.org/10.1016/i.jfinec0.2010.02.009

Chenhall, R. H. (2003). Management control systems design within its organizational context: findings from contingency-based research and directions for the future. Accounting, Organizations and Society, 28(2-3), 127-168. https://doi.org/10.1016/s0361-3682(01)00027-7

Claessens, S., Djankov, S., \& XU, L. C. (2000). Corporate performance in the East Asian financial crisis. The World Bank Research Observer, 15(1), 23-46. https://doi.org/10.1093/wbro/15.1.23

Collins, F., Almer, E. D., \& Mendoza, R. I. (1999). Budget games and effort: differences between the United States and Latin America. Journal of International Accounting, Auditing and Taxation, 8(2), 241-267. https://doi.org/10.1016/S1061-9518/99)00015-4

Collins, F., Holzmann, O., \& Mendoza, R. (1997). Strategy, budgeting, and crisis in Latin America. Accounting, Organizations and Society, 22(7), 669-689. https://doi.org/10.1016/S0361-3682(96)00050-5 
Davila, A., \& Foster, G. (2007). Management control systems in early-stage startup companies. The Accounting $\quad$ Review, 82(4), 937. https://doi.org/10.2308/accr.2007.82.4.907

Fávero, L. P.; Belfiore, P.; Silva, F. L.; Chan, B. L. (2009) Análise de dados Modelagem multivariada para tomada de decisões. Rio de Janeiro: Elsevier.

FIESC. Federação das indústrias do Estado de Santa Catarina. (2017). Disponível em: http://www.fiescnet.com.br/guiawebsc/. Acesso em: 15 de abril de 2017.

Frezatti, F.; Relvas, T. R. S.; Nascimento, A. R. do; Junqueira, E. R.; Bido, D. de S. (2020). Perfil de planejamento e ciclo de vida organizacional nas empresas brasileiras. Revista de Administração, 45(4), 383-399. https://doi.org/10.1016/i.jbusres.2009.11.008

Gordon, L. A., \& Narayanan, V. K. (1984). Management accounting systems, perceived environmental uncertainty and organization structure: an empirical investigation. Accounting, Organizations and Society, 9(1), 33-47. https://doi.org/10.1016/0361-3682(84)90028-X

Hair JR, J. F.; Anderson, R. E.; Tatham, R. L.; Black, W. C. (2005). Análise multivariada de dados. 5. ed. Porto Alegre. Bookman.

Haka, S., \& Krishnan, R. (2005). Budget type and performance-the moderating effect of uncertainty. Australian Accounting Review, 15(35), 3-13. https://doi.org/10.1111/j.1835-2561.2005.tb00247.x

Hayes, D. C. (1977). The contingency theory of managerial accounting. Accounting review, 22-39.

Hopwood, A. G. (2009). The economic crisis and accounting: Implications for the research community. Accounting, Organizations and Society, 34 (6-7), 797-802. https://doi.org/10.1016/j.aos.2009.07.004

IBGE. Instituto Brasileiro de Geografia e Estatística. (2017). Disponível em: http://cnae.ibge.gov.br/busca-online-

cnae.html? view $=$ secao\&tipo $=$ cnae \&versaosubclasse $=9 \&$ versaoclasse $=7 \&$ sec ao $=$. . Acesso em: 23 de janeiro de 2017.

Janke, R., Mahlendorf, M. D., \& Weber, J. (2014). An exploratory study of the reciprocal relationship between interactive use of management control systems and perception of negative external crisis effects. Management Accounting https://doi.org/10.1016/j.mar.2014.01.001

251-270.

Kunc, M., \& Bhandari, R. (2011). Strategic development processes during economic and financial crisis. Management Decision, 49(8), 1343-1353. https://doi.org/10.1108/002517411111163151 
Leite, R. M. Orçamento empresarial: um estudo exploratório em indústria do Estado do Paraná. 2008. 237 f. Dissertação (Mestrado em Contabilidade) Universidade Federal do Paraná - UFPR, Curitiba-PR, 2008.

Miller, D., \& Friesen, P. H. (1982). Innovation in conservative and entrepreneurial firms: Two models of strategic momentum. Strategic management journal, 3(1), 1-25. https://doi.org/10.1002/smj.4250030102

Moores, K., \& Yuen, S. (2001). Management accounting systems and organizational configuration: a life-cycle perspective. Accounting, organizations and society, 26(4-5), 351-389. https://doi.org/10.1016/S03613682(00)00040-4

Neitzke, A. C. A., \& Espejo, M. M. D. S. B. (2016). Estratégia e seus impactos no orçamento empresarial: Um estudo contributivo à tipologia de Porter e ao modelo de Simons. Contabilometria, 3(2).

Otley, D. (2016). The contingency theory of management accounting and control: 1980-2014. Management accounting research, 31, 45-62. https://doi.org/10.1016/j.mar.2016.02.001

Palermo, T., \& Van der Stede, W. (2011). Scenario budgeting: integrating risk and performance. Finance and Management, 184, 10-13.

Panosso, A., Camacho, R. R., Espejo, M. M. D. S. B., \& Abbas, K. (2017). Influência das ferramentas de controle gerencial no desempenho: estudo empírico em empresas industriais paranaenses. Enfoque: Reflexão Contábil, 36(2), 01-17. https://doi.org/10.4025/enfoque.v36i2.33462

Pavlatos, O., \& Kostakis, H. (2018). Management accounting innovations in a time of economic crisis. The Journal of Economic Asymmetries, 18, e00106. https://doi.org/10.1016/j.jeca.2018.e00106

Porter, M. E. (1980). Competitive strategy: Techniques for analyzing industries and competition. New York, 300, 28.

Sandroni, P. (1994). Novo dicionário de economia. $1^{\circ}$ edição. São Paulo: Editora Best Seller.

Schaaper, J., Mizoguchi, S., Nakamura, H., \& Yamashita, S. (2011). Control of French and Japanese subsidiaries in China: implementing control mechanisms before and after the global economic crisis. Asia Pacific business review, 17(4), 411-430. https://doi.org/10.1080/13602381.2011.546626

Staw, B. M., Sandelands, L. E., \& Dutton, J. E. (1981). Threat rigidity effects in organizational behavior: A multilevel analysis. Administrative science quarterly, 501-524. https://doi.org/10.2307/2392337 
Ury, W., \& Smoke, R. Anatomy of a Crisis (1991). Negotiation Theory and Practice/Edited by Breslin JW and Rubin JZ-Cambridge: The Program on Negotiation at Harvard Law School.

Van der Stede, W. A. (2011). Management accounting research in the wake of the crisis: some reflections. European Accounting Review, 20(4), 605-623. https://doi.org/10.1080/09638180.2011.627678

Van der Stede, W. A. (2014). A manipulationist view of causality in cross-sectional survey research. Accounting, Organizations and Society, 39(7), 567-574. https://doi.org/10.1016/j.aos.2013.12.001

Waal, A., \& Mollema, E. (2010). Six courses of action to survive and thrive in a crisis. Business Strategy Series, $11(5), \quad 333-339$. https://doi.org/10.1108/17515631011080740

Waterhouse, J. H., \& Tiessen, P. (1978). A contingency framework for management accounting systems research. Accounting, Organizations and Society, 3(1), 6576. https://doi.org/10.1016/0361-3682(78)90007-7

Welsch, G. A. (1983). Orçamento Empresarial. Tradução e Adaptação: Antônio Zoratto Sanvicente. São Paulo: Atlas.

Weston, J. F., \& Brigham, E. F. (2000). Fundamentos da administração financeira. $10^{a}$ edição. Makron.

Zona, F. (2012). Corporate investing as a response to economic downturn: Prospect theory, the behavioural agency model and the role of financial slack. British Journal of Management, 23(S1). https://doi.org/10.1111/i.14678551.2012.00818.x 


\section{CONTRIBUIÇÕES DOS AUTORES}

\begin{tabular}{|l|c|c|c|}
\hline \multicolumn{1}{|c|}{ Contribuição } & Ângela Bilk & $\begin{array}{c}\text { Marcia } \\
\text { Zanievicz da } \\
\text { Silva }\end{array}$ & $\begin{array}{c}\text { Carlos Eduardo } \\
\text { Facin Lavarda }\end{array}$ \\
\hline $\begin{array}{l}\text { 1. Idealização e concepção do assunto } \\
\text { e tema da pesquisa }\end{array}$ & $\checkmark$ & $\checkmark$ & \\
\hline 2. Definição do problema de pesquisa & $\checkmark$ & $\checkmark$ & \\
\hline $\begin{array}{l}\text { 3. Desenvolvimento da Plataforma } \\
\text { Teórica }\end{array}$ & $\checkmark$ & $\checkmark$ & $\checkmark$ \\
\hline $\begin{array}{l}\text { 4. Delineamento da abordagem } \\
\text { metodológica da pesquisa }\end{array}$ & $\checkmark$ & $\checkmark$ & $\checkmark$ \\
\hline 5. Coleta de dados & $\checkmark$ & $\checkmark$ & $\checkmark$ \\
\hline $\begin{array}{l}\text { 6. Análises e interpretações dos dados } \\
\text { coletados }\end{array}$ & $\checkmark$ & $\checkmark$ & $\checkmark$ \\
\hline 7. Conclusões da pesquisa & $\checkmark$ & $\checkmark$ & $\checkmark$ \\
\hline 8. Revisão crítica do manuscrito & $\checkmark$ & $\checkmark$ & \\
\hline $\begin{array}{l}\text { 9. Redação final do manuscrito, } \\
\text { conforme as normas estabelecidas pela } \\
\text { Revista. }\end{array}$ & & $\checkmark$ & \\
\hline 10. Orientação & & $\checkmark$ & \\
\hline
\end{tabular}

